\title{
Modeling Time in Medical Education Research: The Potential of New Flexible Parametric Methods of Survival Analysis
}

\author{
Gilbert Reibnegger \\ Institute of Physiological Chemistry, Center of Physiological Chemistry, Medical University of Graz, \\ Graz, Austria \\ Email: gilbert.reibnegger@medunigraz.at
}

Received September $5^{\text {th }}, 2012$; revised October $4^{\text {th }}, 2012$; accepted October $20^{\text {th }}, 2012$

\begin{abstract}
Time - the duration of a certain process or the timing of a specified event - plays a central role in many situations in medical research. Waiting time analysis ("survival analysis") is a field of statistics providing the tools for solving the unique problems of such studies. In particular, waiting time analysis correctly handles the typical positively skewed distributions of waiting times as well as censored observations on study subjects for whom the target event does not occur before data collection ends. For decades, non-parametric Kaplan-Meier analysis and semiparametric Cox regression despite some inherent limitations have dominated waiting time analysis in medical contexts, while parametric models, although in principle offering important theoretical advantages, were scarcely applied in practice because of lacking flexibility. Recently, however, new flexible parametric methods (Royston-Parmar models) became available offering exciting new research potential. Surprisingly, although medical education research deals with a range of typical problems suited for waiting time analysis, the methods were rarely used in the past. By re-analyzing data from a previous investigation on study dropout of medical students, this is the first study demonstrating the usefulness and practical applications of waiting time analysis with special emphasis on Royston-Parmar models in a medical education research environment.
\end{abstract}

Keywords: Study Dropout; Medical Studies; Survival Analysis; Flexible Parametric Models; Royston-Parmar Models

\section{Introduction}

Survival analysis represents a field of statistics extensively used by medical researchers. Survival analysis deals with survival times of groups of individuals and enables qualitative identification and quantitative estimation of single or multiple predictive factors influencing and modulating survival times. Particularly in fields like oncology or chronic diseases medical literature employing this specialized bundle of statistical methods is abundant.

In medical education research, survival analysis or-more general- "analysis of waiting times" appears promising since also in this research field time frequently plays an important role. Data on students or learners in general may be time-dependent, and quite often, time itself can be viewed as a key variable in an investigation. For example, a central observation of a research study could be for each individual student included into the analysis, the time span elapsing from one welldefined starting event (for example, her or his beginning of a whole study program or a specified course) to another welldefined terminating event (for example, graduation, attaining a well-defined competency, passing an examination, or even study dropout). Such time intervals are generally called "waiting times" and I shall use the terms "survival analysis" and "analysis of waiting times" synonymously in this paper.

Somewhat surprisingly, however, the powerful techniques of waiting time analysis have been scarcely exploited in medical education research literature. For example, in a recent literature review on study dropout in medical studies (O'Neill et al.,
2011), 13 quality-assessed scientific articles were analyzed in depth, but in none of these papers the techniques of waiting time analysis were employed although, of course, the waiting times from study start to study dropout expectably could have been a natural issue of concern of such investigations. Rather, the authors of the various papers cited used variants of linear or logistic regression, analysis of frequency tables and other more traditional statistical procedures, thus abandoning full exploitation of an important source of potential information.

During the past decades, analysis of waiting times/survival analysis was nearly exclusively dominated by two methods, namely, the nonparametric product-limit approach (Kaplan \& Meier, 1958) (also termed "Kaplan-Meier"-method) and the semiparametric proportional hazards model (Cox, 1972) (also called "Cox"-regression). Parametric methods (Brown, 1982), despite their theoretical superiority, were rarely employed due to their notorious lack of flexibility to fit real life data sets sufficiently well. Readers interested in the mathematical details of such "classical" parametric models (as I shall call these older models in due course) are referred to a comprehensive monograph (Cleves et al., 2008).

In the past decade, however, new flexible parametric methods ("Royston-Parmar" models, RP models) were developed (Royston \& Parmar, 2002) enabling researchers to exploit the full potential of survival analysis. These techniques allowapparently for a very broad variety of practical situations - the estimation of smooth time-dependent survival curves and hazard functions, as well as many time-dependent quantities derived from these, in a most appealing and instructive way, to- 
gether with estimates of their uncertainties and confidence limits. Employing up-to-date computing strategies involving cubic spline functions, RP models overcome the problems of poor fit of classical parametric methods and go beyond the traditional approaches of the Kaplan-Meier and the Cox techniques which provide only pointwisely defined - and hence "noisy"- estimates of the survival and hazard functions.

With this paper I intend to call the attention of medical education researchers to the riches of analysis of waiting times in general, and of the new RP techniques in particular. While traditional analysis of waiting times, albeit infrequently, has been used in medical education research (De Champlain et al., 2006; Reibnegger et al., 2010, 2011; Ries et al., 2009), to the best of my knowledge this is the first paper employing the new flexible RP methods in the field of medical education research. To illustrate the potential of the new methods I reanalyze previously published data on study dropout of medical students at the Medical University of Graz before and after the installation of a selective admission procedure instead of open admission (Reibnegger et al., 2011).

\section{Materials and Methods}

In a previous paper (Reibnegger et al., 2011) we presented data on study dropout of all 2860 medical students who were admitted to human medicine diploma program at the Medical University of Graz in the academic years 2002/03 until 2008/09. Data collection was closed by end of February, 2010. During the years $2002 / 03$ to $2004 / 05$, admission to the study program was open to all applicants who had passed secondary school; since 2005/06, however, study places were restricted and applicants were admitted only after passing an admission procedure based on a knowledge test. There were $1630(57.0 \%)$ women and $1230(43.0 \%$ men); age range at study entry was from 17.51 to 50.03 years with a median of 19.69 years and an interquartile range from 18.92 to 20.89 years. Besides absence versus presence of an admission test, in this paper I shall analyze the variables sex and age at study entry for their potential as additional explanatory factors for the probability of retention within study versus study dropout. As in the earlier paper (Reibnegger et al., 2011) I dichotomize age at the third quartile of 20.89 years.

In the earlier paper, we analyzed the data by the traditional approach using nonparametric Kaplan-Meier estimation of "survival" curves (which in this particular setting means "probability of retention within the study program") and by the Cox proportional hazards approach in order to identify predictive variables and to quantify their multiplicative effect on the dropout hazard of students. Briefly, we found open admission versus active selection of students to be an extraordinarily strong predictor of study dropout; while 764 of 1971 (38.8\%) openly admitted students dropped out from study during the observation period, the respective fraction in selected students was only 41 of $889(4.6 \%)$. Additionally, while in the selected students sex and age at study entry were of no predictive significance, both variables were significant predictors for study dropout in openly admitted students: women were at higher risk of dropout, and so were students aged above the threshold of 20.89 years.

In the present paper, I demonstrate the usefulness of the RP models by re-analyzing these data for two research questions:

- the impact of the installation of a selective admission pro- cedure in 2005/06.

- the prognostic strengths of the two explanatory variables sex and age at study entry in the cohorts openly admitted to the study program from 2002/03 to 2004/05.

As RP models use cubic spline functions (Royston \& Parmar, 2002) for a flexible fit of the data, one can vary the number of such spline functions ("degrees of freedom") included in the models. Thus, one can compare the suitability of RP models with different numbers of degrees of freedom to fit the observed data. Furthermore, RP models allow going beyond the proportional hazards assumption; non-proportional hazards models are easily obtained, and so are models with different scaling such as proportional odds models (Royston \& Lambert, 2011). To demonstrate the superiority of RP models over classical parametric models, I compare different RP models and a range of classical parametric models for their ability to fit the data. For final model selection, I employ the Akaike information criterion (Akaike, 1974) (AIC). This widely used model selection criterion computes the deviance of the fit:

$$
\mathrm{AIC}=2 k-2 \times \log _{e} L
$$

where $k$ is the dimension of the model (i.e., the number of fitted parameters) and $L$ is the maximized likelihood function for the estimated model. Generally, models with smaller AIC are considered to fit the data better.

For all statistical analyses, I use commercially available software (Stata Statistical Software: Release 11. StataCorp, 2009, College Station, TX, USA); this software package presently appears to provide the largest variety of survival models including classical parametric models (Cleves et al., 2008) as well as flexible RP models (Royston \& Lambert, 2011).

\section{Results}

\section{Impact of an Active Admission Procedure}

Table 1 shows the results of a search among six classical parametric models with different degree of complexity and four different RP models, for their abilities to fit the data. As expected, the least suitable model is the simple exponential model assuming a constant, time-independent hazard; it has a particularly low flexibility and consequently shows the largest value of AIC. Of the classical models, the Gompertz model provides the best fit of the data. However, all RP models tested outperform the classical models markedly. Among the studied models, the proportional odds model with 4 degrees of freedom is identified as the most suitable. (I could easily have tested more complex RP models with potentially even better fit, but I deliberately restricted the analysis to these few models, since fit of the data is already excellent, and the advantages of the new techniques can readily be demonstrated.)

Figure 1 visualizes the meaning of the abstract AIC values of the various models with respect to the curves for cumulative retention probabilities and hazard rates. While for the group of students admitted after an admission test, the curves for retention probability do not vary greatly among the models used, it is obvious from Figure 1(a) that for the cumulative retention probability curve of the openly admitted students the exponential model shows a very poor fit. The best-fitting model among the classical parametric models, i.e., the Gompertz model, shows quite an improvement, but as can be seen, the proportional odds RP model follows nearly exactly the non-parametric 


\section{G. REIBNEGGER}

Table 1.

Model selection for analyzing the impact of absence versus presence of a selective admission procedure. ${ }^{\mathrm{a}} \mathrm{AIC}$, Akaike information criterion; ${ }^{b}$ The models are either on the proportional hazards $(\mathrm{PH})$ or proportional odds (PO) scale with 2 or 4 degrees of freedom as defined by the numbers in parenthesis.

\begin{tabular}{cccc}
\hline & log likelihood & Model dimension & $\mathrm{AIC}^{\mathrm{a}}$ \\
\hline Classical models & & & \\
\hline Exponential & -2615.371 & 2 & 5234.742 \\
Weibull & -2578.268 & 3 & 5162.536 \\
Gompertz & -2491.614 & 3 & 4989.227 \\
Lognormal & -2521.288 & 3 & 5048.576 \\
$\begin{array}{c}\text { Loglogistic } \\
\text { Generalized } \\
\text { gamma }\end{array}$ & -2548.806 & 3 & 5103.612 \\
\hline $\begin{array}{c}\text { Royston-Parmar } \\
\text { models }\end{array}$ & -2514.002 & 4 & 5036.005 \\
\hline PH(2) & -2470.833 & 4 & 4949.667 \\
PH(4) & -2410.608 & 6 & 4833.216 \\
PO(2) & -2471.954 & 4 & 4951.907 \\
PO(4) & -2409.230 & 6 & 4830.460 \\
\hline
\end{tabular}

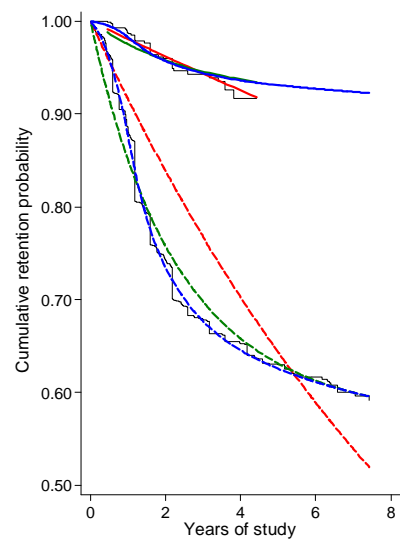

(a)

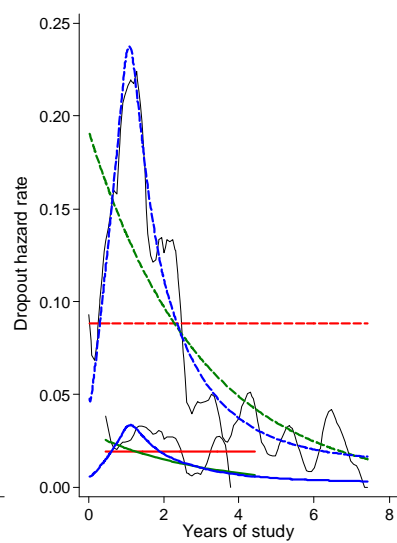

(b)
Figure 1.

Dropout behavior of openly admitted versus selected students-a comparison of different models. (a) Cumulative retention probabilities. Shown are three parametric models: exponential model (red), Gompertz model (green) and proportional odds RP model with 4 degrees of freedom (blue). Solid lines denote selected students; dashed lines denote openly admitted students. For comparison, also the Kaplan-Meier estimates are shown (thin black step lines); (b) Dropout hazard rates. Shown are the results from three parametric models: exponential model (red), Gompertz model (green) and proportional odds RP model with 4 degrees of freedom (blue). Solid lines denote selected students; dashed lines denote openly admitted students. For comparison, also the nonparametric smoothed kernel estimates are shown (thin black wiggly lines).

Kaplan-Meier estimates. The differences between the models become even better evident, however, when we look at the hazard rates (Figure 1(b)): the exponential model has fixed, time-constant hazard rates for the two groups which are graphi- cally represented by the horizontal straight lines in Figure 1(b). The Gompertz model is restricted to monotonically rising oras in our example - falling hazard rates, and it is therefore obviously unable to reflect the true time course of the hazard rates. The only model describing the nature of the hazard functions for this data set adequately is the RP model; for the openly admitted students, it clearly reveals the initial steep dropout hazard rate increase leading to a sharp peak between 1 and 2 years of study and the marked decrease thereafter. For comparison, a nonparametric estimate of the hazard functions obtained by a kernel technique (Muller \& Wang, 1994) (thin irregular lines in Figure 1(b)) is shown. These estimates according to the pointwise nature of the nonparametric method are generally known to show a number of artificial wiggles and irregularities which are probably of no real significance (Royston \& Parmar, 2011). However, the general non-monotonic character of the hazard rates and the marked peak between 1 and 2 years of study is obvious also from these curves. Summarizing, the major weakness of the classical parametric methods, namely, their notorious inflexibility, as well as the high flexibility and thus, superior fitting potential, of the RP models become evident from the figure.

As we have reported in our previous paper (Reibnegger et al., 2011), the difference between the retention probability curves of openly admitted versus selected students is highly significant: the Breslow test (Breslow, 1970) yields a chi square $\chi^{2}=$ 200.24 (1 degree of freedom) for the difference between the two Kaplan-Meier curves, and the RP estimate for the regression coefficient for the variable "open versus selective admission" is 2.089 with a standard error $\mathrm{SE}=0.167$, yielding a standardized normal deviate $\mathrm{Z}=2.089 / 0.167=12.50$; both statistics being highly significant $(P \leq 0.0001)$.

Beyond visualization of retention probabilities and hazard rates, Figure 2 shows an array of diverse post-estimation results which were obtained and visualized after fitting the RP proportional odds-model with 4 degrees of freedom. Figure 2(a) depicts the time-dependent difference between the cumulative retention probabilities of selected students minus that of openly admitted students, together with its $95 \%$ confidence interval (c.i.). Initially, the difference function shows - as a natural consequence of the initially steeply increasing dropout hazard rate for openly admitted students - a steep increase, whereas after 2 years of study, the difference function becomes markedly flatter.

Figure 2(b) serves to explain the central feature distinguishing a proportional odds model from the much better known proportional hazards models. Here, for both student populations (open versus selective admission), I have depicted the natural logarithms of the odds (the so-called "logits") of the cumulative retention probability functions: at any time $t$, the logit of the cumulative retention probability function $S(t)$ is given by

$$
\operatorname{logit}[S(t)]=\log _{e} \frac{S(t)}{1-S(t)} .
$$

The proportional odds model assumes that the ratio between the odds of two retention functions (or, equivalently, the difference between the respective logits) is constant over time. As can be seen from Figure 2(b), the two logit functions over the whole time range have a constant vertical distance. Actually, this distance is by definition numerically determined by the regression coefficient which in our example is 2.089. An alter 


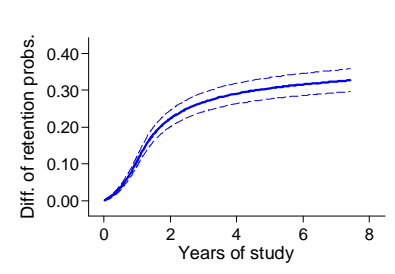

(a)

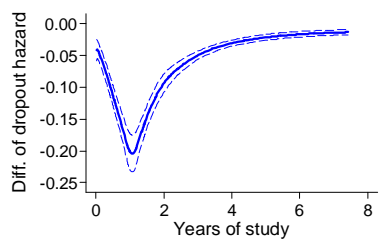

(c)

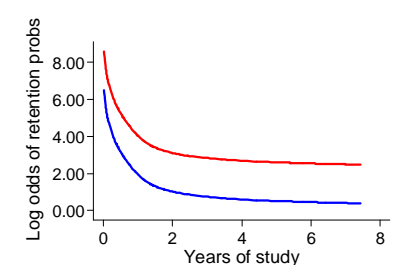

(b)

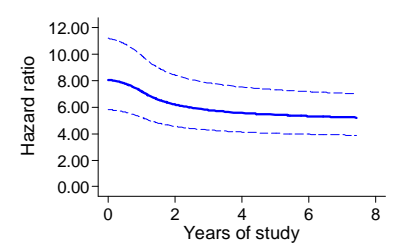

(d)
Figure 2.

Dropout behavior of openly admitted versus selected students-additional results derived from the proportional odds RP model with 4 degrees of freedom. (a) Difference function of the cumulative retention probabilities (selected students minus openly admitted students). The solid line represents the estimated difference function; the dashed lines denote the $95 \%$ c.i.; (b) Logit functions (natural logarithms of the odds of retention) for openly admitted students (red) and for selected students (blue). Note the constant distance between the blue and the red curves; this distance is just equal to the respective regression coefficient of 2.089, irrespective of time (see text); (c) Difference function of the dropout hazard rates (selected minus openly admitted students. The solid line represents the estimated difference function; the dashed lines denote the $95 \%$ c.i.; (d) The dropout hazard ratio (numerator: hazard rate of openly admitted students; denominator: hazard rate of selected students). The solid line represents the estimated hazard ratio; the dashed lines denote the $95 \%$ c.i.

native interpretation of the regression coefficient would be to say that, at any time, the odds of retention within study of students having passed the admission test are higher than the retention odds of an openly admitted student by a factor given by

$$
\mathrm{e}^{2.089}=8.1
$$

The bottom row of Figure 2 deals with functions of hazard rates. The difference between the hazard function of selected minus that of openly admitted students (Figure 2(c)) exhibits a (negative) peak during the initial time period, and decreases in absolute value during the subsequent course of study, finally approaching values near zero. Thus, even in the high dropout risk group of openly admitted students, "surviving" within the study program longer than the critical interval of about two years strongly reduces the dropout risk and, in turn, increases the probability for final graduation.

Figure 2(d) shows the time-dependent behavior of the hazard ratio: in the predominant "culture" of the semiparametric Cox model taking for granted the proportional hazards assumption, this function would - by definition - be a constant not depending on time. (Actually, a Cox regression model yields a constant hazard ratio of 6.89 with a $95 \%$ c.i. ranging from 5.03 to 9.45 ). According to the proportional odds model used here, however, the hazard ratio is no longer forced to be constant but is allowed to depend on time; it is higher in the initial phase and thereafter decreases. Typically, proportional odds models are particularly well suited to describe situations with converging hazards; so the behavior of the hazard ratio over time depicted in Figure 2(d) appears very reasonable. Obviously, the Cox regression enforcing a time-independent hazard ratio, with 6.89 yields just an average value. The interpretation of the hazard ratio is straightforward; by the Cox model, the dropout risk of an openly admitted student is 6.89 times higher than that of an actively selected student, irrespective of time. In contrast, the probably more realistic Royston-Parmar model based on the proportional odds assumption indicates that the ratio between the dropout risk of an openly admitted student compared to that of a selected fellow student is about 8 in the initial phase, and decreases to values below 6 during the later phases of study. It is imperative, however, to bear in mind that despite these values for the ratio of the hazard rates of both student populations, the absolute difference between the dropout hazard rates according to Figure 2(c) nearly vanishes in the later phase of the study program.

\section{Impact of Sex and Age in Openly Admitted Students}

A model selection run was performed for this second problem in an analogous fashion as reported in Table $\mathbf{1}$ (details not shown). Here, a proportional hazards model with 4 degrees of freedom was identified as the optimum model according to the AIC.

Following this optimum model, the dropout hazard ratio (which now is held constant over time according to the proportional hazards assumption) for sex is 1.70 with a $95 \%$ c.i. ranging from 1.46 to 1.98 . That means that women have a dropout risk 1.70 times higher than that of men. Likewise, students with ages at study entry above 20.89 years carry a 2.02 times higher dropout hazard ( $95 \%$ c.i. from 1.73 to 2.43$)$ than their younger fellow students.

A comparable Cox regression model yields essentially the same hazard ratios: for sex, the hazard ratio is $1.69(95 \%$ c.i. from 1.45 to 1.97 ), and for age, it is 1.99 (95\% c.i. from 1.70 to 2.33). So obviously both techniques agree nearly perfectly regarding the determination of hazard ratios; the Cox regression technique, however, is clearly inferior regarding the estimation of smooth retention or hazard functions.

Figure 3 visualizes some results that can be drawn from the analysis: Figure 3(a) shows the time course of the retention probabilities for the four students groups defined by the binary variables sex (women versus men) and age (younger versus older than 20.89 years). Again, the comparison with the nonparametric Kaplan-Meier estimates shows an excellent agreement. It is remarkable how strong the dropout risk differs among the four student groups. Figure 3(b) represents the time-dependent courses of the dropout hazard rates for the four students groups: after a steep increase of dropout hazard within the first year of study with a peak early in the second year, the dropout hazards then decrease markedly. Note that the four hazard rates are, at each time point, strictly proportional to each other as a consequence of the proportional hazards model used: the lowest dropout risk is associated with men below 20.89 years. At any specified time point, the dropout hazard of men above that age threshold is 2.02 times higher. Similarly, the dropout hazard of women below 20.89 years is 1.70 times higher than that of young men at any given time point, and for women older than 20.89 years (the highest-risk group), the dropout hazard - at any fixed time point - exceeds the hazard of young men by a factor given as the product $1.70 \times 2.02=$ 3.43 .

Figure 3(c) shows the difference of the hazard rates between 


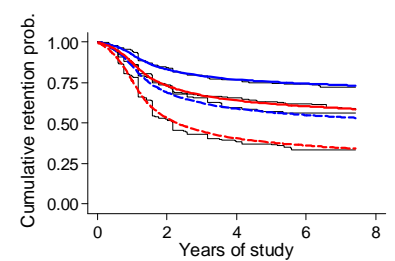

(a)

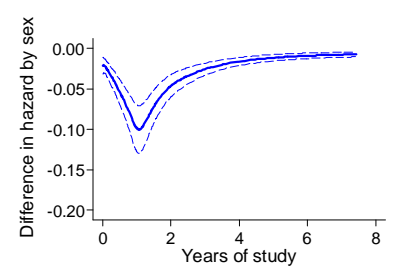

(c)

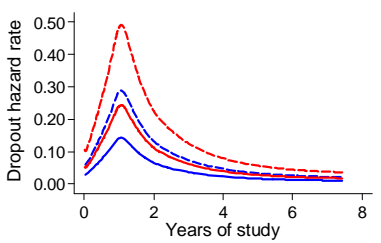

(b)

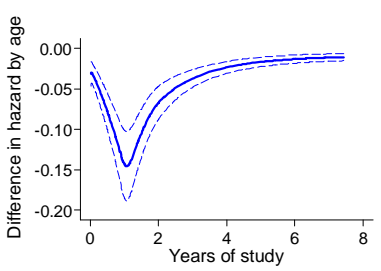

(d)
Figure 3.

Dropout behavior of openly admitted students grouped by sex and age at study entry (below versus above 20.89 years) - results of a proportional hazards RP model with 4 degrees of freedom. (a) Cumulative retention probabilities of men below (solid blue line) and above 20.89 years (dashed blue line) and of women below (solid red line) and 20.89 years (dashed red line). For comparison, also the Kaplan-Meier estimates (thin black step lines) are included for the four student groups; (b) Dropout hazard rates of men below (solid blue line) and above 20.89 years (dashed blue line) and of women below (solid red line) and 20.89 years (dashed red line); (c) Difference function of the dropout hazard rates by sex (men minus women). The solid line represents the estimated difference function; the dashed lines denote the $95 \%$ c.i.; (d) Difference function of the dropout hazard rates by age at study entry (below minus above 20.89 years). The solid line represents the estimated difference function; the dashed lines denote the $95 \%$ c.i.

men and women, and Figure 3(d) demonstrates the hazard difference between younger and older students as a function of time. Clearly, other quantities and comparisons could be added in an analogous fashion as in Figure 2; the potential of the new flexible parametric methods for analysis of waiting times, however, appears to be sufficiently demonstrated by the examples given.

\section{Discussion}

There are some deficits known of the Kaplan-Meier and the Cox approach. First, Kaplan-Meier as well as Cox estimates of the survivor function can be calculated only at those time points when actually at least one study subject experiences the terminating event. Between such time points, the survivor function is indeterminate. Moreover, these estimates of the survivor function are highly serially correlated; therefore, the resulting step functions often display an undulating appearance moving away from and back toward the general trend. Second, because of the pointwise nature of the survivor function there is no easy way to calculate the hazard function: functions defined only at discrete time points cannot be differentiated with respect to time in an easy way. There are numerical approximations available using kernel algorithms (Muller \& Wang, 1994) but the resulting estimates of smoothed hazard functions are not really satisfactory for many purposes (Royston \& Parmar, 2011). (A kernel smoother is a statistical technique for estimating a real valued function by using its noisy observations, when no parametric model for this function is known.) The smoothed hazard functions depend strongly on the kernel algorithm actually used, and, as Figure 1(b) shows, they typically tend to exhibit artificial wiggles without real significance (Royston \& Parmar, 2011). The semiparametric Cox regression technique (Cox, 1972) has its major strengths in estimating the multiplicative effects of one or more explanatory variables of different scale qualities (interval, ordinal, and nominal) on the hazard function. The Cox method shares with the Kaplan-Meier approach not only the inability of estimating continuous and analytic survivor curves but also the "blindness" with regard to the actual time course of the baseline hazard function. "The success of Cox regression has perhaps had the unintended side-effect that practitioners too seldomly invest efforts in studying the baseline hazard... A parametric version [of the Cox model], ... if found to be adequate, would lead to more precise estimation of survival probabilities and ... concurrently contribute to a better understanding of the phenomenon under study" (Hjort, 1992).

In principle, parametric models for waiting time analysis would provide a straightforward solution to the inability of both the Kaplan-Meier and the Cox model to produce smooth and analytic survivor and hazard functions. Various parametric models exist since decades; these classical parametric models differ from each other because of different assumptions regarding the probability distribution function underlying the observed waiting times. The simplest classical parametric model is the exponential model assuming a hazard function which is held constant over time. The Weibull model and the Gompertz model have a hazard function either monotonically rising or monotonically falling over time. More complex models, such as the lognormal, the log-logistic and the generalized Gamma model, allow greater flexibility in modeling hazard functions exhibiting turning points: the hazard, e.g., may be rising at earlier times and, after going through a maximum at a specified time, falling thereafter. Detailed descriptions of these models are available (Cleves et al., 2008).

In theory, classical parametric models overcome the deficiencies of the Kaplan-Meier and the Cox methods. They provide smooth, analytic estimates for the hazard and survivor functions, and they are able to include, like the Cox model, any combination of predictor variables. They allow easy computation of any type of output, for example, time-dependent survivor or hazard functions as well as time-dependent difference functions and hazard ratios, and they provide the possibility to calculate standard estimation errors and confidence intervals for all these interesting quantities. Why then were these models hardly used in medical research? The answer is simple: real life waiting times data usually do not follow the theoretical distribution functions underlying the models sufficiently well, and so the major problem with classical parametric models is simply lack of fit because of limited flexibility.

Therefore, the new flexible parametric RP models developed during the past decade provide a real step forward because they retain the theoretical strengths of classical parametric models and at the same time, overcome their limited flexibility. Briefly, these methods make use of cubic spline functions providing the models with sufficient flexibility to adequately fit real life data sets (Andersson et al., 2011; Colzani et al., 2011; Nelson et al., 2007). The models are accessible in one of the major commercial statistical software packages (Royston \& Parmar, 2011).

RP models not only show improved ability to fit real life data sets due to the use of cubic spline functions, the number and properties of which can be easily controlled and modified, they 
also go beyond the traditional proportional hazards assumption (which, as this paper demonstrates, is not necessarily the best choice) by allowing the user to choose other scales, such as, e.g., the proportional odds scale or the probit (inverse normal probability) scale. Clearly, the greatly increased number of possible modeling options requires methods for model selection; the Akaike information criterion AIC (Akaike, 1974) is a useful tool for that purpose. (An alternative to the AIC is the Bayes information criterion BIC (Schwarz, 1978).)

By re-analyzing published study dropout data before and after the installation of an admission test for medical students (Reibnegger et al., 2011) I have addressed two different questions using RP models. In both cases, I have compared various RP models with six classical parametric models of increasing complexity. Model comparison using the AIC shows the marked improvement of model fit when switching from classical to RP models. Interestingly, for the first study question, namely, the impact of the absence versus presence of an admission test on study dropout, a proportional odds RP model with 4 degrees of freedom was found to fit the data even better than a comparable proportional hazards model, indicating that the dropout hazard rates of both student groups converge in the long run (Bennett, 1983). The graphical comparison of different models (Figure 1) shows that the simple exponential model is clearly not suitable to fit the retention probabilities. Among the other classical parametric models, the Gompertz model was identified to provide the best fit, and actually its deviation from the Kaplan-Meier estimates apparently is not very large. However, the inspection of the hazard rates then provides very convincing evidence that none of the classical models sufficiently represents the true time dependence of the hazard functions. The exponential model is restricted to constant hazard rates; it is, therefore, completely unable to follow the observed course of the hazard which strongly increases in the early phase, peaks between year 1 and year 2, and rapidly decreases afterwards. Also the Gompertz model is not satisfactory; according to its restriction to monotonically increasing or falling hazard functions, it is unable to reflect the true nature of the hazard functions. In strong contrast, the RP model easily catches the true course of the hazard functions, while at the same time avoiding the noisy and wiggly structure of the nonparametric kernel estimates.

Parametric models adequately fitting the observed data can be exploited to yield much more detailed information than nonparametric or semiparametric methods. In contrast to the latter, differences or ratios between survivor probabilities as well as between hazard rates of different groups of individuals are easily obtainable, and so are the confidence intervals of these quantities. Additionally, many other functions derived mathematically from the central quantities (cumulative survivor probability and hazard rate) are accessible, such as, e.g. the logits (see Figure 2 for examples).

Secondly, the effects of predictor variables sex and age on cumulative retention probabilities as well as on dropout hazards were studied in the cohorts of openly admitted students. Again, I have compared classical as well as RP parametric models for their suitability to describe these data. Here, a proportional hazards RP model could be identified as best-fitting model. The excellent fit of the resulting retention probabilities is obvious from Figure 3(a). According to the nature of this model, the hazard functions of the four student groups by sex and age in this case are strictly proportional, and hazard ratios between different student groups are therefore constant over time. Notably, the hazard ratios obtained for the two predictor variables sex and age are nearly identical to those obtained by a Cox regression technique. As shown in the Results section, also the interpretation of these hazard ratios as well as their multiplicative application is identical to the Cox model.

What the Cox model fails to provide, however, is the detailed time course of the hazard rates and of, e.g., the difference functions between different student groups. The Cox model yields practically the same information regarding the strengths and the uncertainties of the constant hazard ratios; it does not, however, point out clearly enough that the absolute differences between the hazard functions of men versus women, or of younger versus older students, after a strong peak in the initial phase of the study program nearly vanish in the long run. It should be noted that this important feature is also not easily grasped from Kaplan-Meier curves: depicting the cumulative retention (survival) probabilities, in our example these curves are strongly determined by the marked initial differences between the hazard functions. It is mainly within the initial two years that the cumulative plots become separated, and these separations remain visible despite vanishing differences of hazard rates in the later years. Thus, the cumulative probability curves visually suggest a marked difference between the different student groups particularly at later times; however, as the hazard rates of all groups nearly vanish at later times, a more appropriate impression of what really happens is only provided by inspection of the hazard rates themselves. In conclusion, straightforwardly drawing the time-dependent differences between the hazard functions, which is easily accomplished after estimating a RP model, explicates the intimate details of the time-dependent hazard rates in a much better way.

An important practical issue for usage of a new statistical technique by a broad scientific community is the availability of user-friendly computing software. Kaplan-Meier curves and Cox models as well as classical parametric models can be obtained by several commercially available software packages like SAS, STATA and SPSS. Practical examples including the commands required for performing typical analyses by means of these mentioned packages are available (Kleinbaum \& Klein, 2005). The new flexible RP models can be fitted and analyzed by STATA Software which to my knowledge presently is the only commercial package allowing convenient application of these new techniques. A recent monograph (Royston \& Lambert, 2011) presents numerous examples including all the STATA commands required for even very advanced computations and for graphical visualization of the results.

\section{Conclusion}

The new generation of flexible parametric RP models for analysis of waiting times opens new horizons to investigate time-dependent phenomena. The models overcome limitations of the popular Cox regression method by enabling much more detailed insight into the time course of hazard rates. Moreover, being flexible extensions of classical parametric models, they share the theoretical advantages of the latter while overcoming their limited applicability due to lack of flexibility. In medical education research, one could imagine many research questions that could be studied in greater depth than before by such models: proper modeling of the time intervals required by students to attain defined study goals and investigating the detailed in- 


\section{G. REIBNEGGER}

fluences of potential predictor variables by these potent methods could strongly enhance the spectrum of research directions.

\section{REFERENCES}

Akaike, H. (1974). A new look at the statistical model identification. IEEE Transactions on Automatic Control, 19, 716-723. doi:10.1109/TAC.1974.1100705

Andersson, T. M. L., Dickman, P. W., Eloranta, S., \& Lambert, P. C. (2011). Estimating and modeling cure in population-based cancer studies within the framework of flexible parametric survival models. BMC Medical Research Methodology, 11, 96. doi:10.1186/1471-2288-11-96

Bennett, S. (1983). Analysis of survival data by the proportional odds model. Statistics in Medicine, 2, 273-277. doi:10.1002/sim.4780020223

Breslow, N. (1970). A generalized Kruskal-Wallis test for comparing K samples subject to unequal patterns of censorship. Biometrika, 57, 579-594. doi:10.1093/biomet/57.3.579

Brown, B. W. Jr. (1982). Estimation in survival analysis: Parametric models, product-limit and life-table methods. In V. Miké, \& K. E. Stanley (Eds.), Statistics in medical research. Methods and issues, with applications in cancer research (pp. 317-339). New York, Chichester, Brisbane, Toronto, Singapore: John Wiley and Sons.

Cleves, M., Gutierrez, R., Gould, W., \& Marchenko, Y. (2008). An introduction to survival analysis using stata (2nd ed.). College Station, TX: StataCorp LP., Stata Press.

Colzani, E., Liljegren, A., Johansson, A. L. V., Adolfsson, J., Hellborg, H., Hall, P. F. L., \& Czene, K. (2011). Prognosis of patients with breast cancer: Causes of death and effects of time since diagnosis, age, and tumor characteristics. Journal of Clinical Oncology, 29, 40144021. doi:10.1200/JCO.2010.32.6462

Cox, D. R. (1972). Regression models and life tables (with discussion). Journal of the Royal Statistical Society, 34, 187-220.

De Champlain, A., Sample, L., Dillon, G. F., \& Boulet, J. R. (2006). Modeling longitudinal performances on the United States Medical Licensing Examination and the impact of sociodemographic covariates: An application of survival data analysis. Academic Medicine, 81, S108-S111. doi:10.1097/00001888-200610001-00027

Hjort, N. L. (1992). On inference in parametric survival data models.
International Statistical Review, 60, 355-387. doi:10.2307/1403683

Kaplan, E. L., \& Meier, P. (1958). Nonparametric estimation from incomplete observations. Journal of the American Statistical Association, 53, 457-481. doi:10.1080/01621459.1958.10501452

Kleinbaum, D. G., \& Klein, M. (2005). Survival analysis: A self-learning text (2nd ed.). New York: Springer Science, Business Media, LLC.

Muller, H.-G., \& Wang, J.-L. (1994). Hazard rate estimation under random censoring with varying kernels and bandwidths. Biometrics, 50, 61-76. doi: $10.2307 / 2533197$

Nelson, C. P., Lambert, P. C., Suire, I. B., \& Jones, D. R. (2007). Flexible parametric models for relative survival, with application in coronary heart disease. Statistics in Medicine, 26, 5486-5498. doi: $10.1002 / \operatorname{sim} .3064$

O’Neill, L. D., Wallstedt, B., Eika, B., \& Hartvigsen, J. (2011). Factors associated with dropout in medical education: A literature review. Medical Education, 45, 440-454. doi:10.1111/j.1365-2923.2010.03898.x

Reibnegger, G., Caluba, H.-C., Ithaler, D., Manhal, S., Neges, H. M., \& Smolle, J. (2010). Progress of medical students after open admission or admission based on knowledge tests. Medical Education, 44, 205 214. doi:10.1111/j.1365-2923.2009.03576.x

Reibnegger, G., Caluba, H.-C., Ithaler, D., Manhal, S., Neges, H. M., \& Smolle, J. (2011). Dropout rates in medical students at one school before and after the installation of admission tests in Austria. Academic Medicine, 86, 1040-1048. doi:10.1097/ACM.0b013e3182223a1b

Ries, A., Wingard, D., Morgan, C., Farrell, E., Letter, S., \& Reznik, V. (2009). Retention of junior faculty in academic medicine at the University of California, San Diego. Academic Medicine, 84, 37-41. doi:10.1097/ACM.0b013e3181901174

Royston, P., \& Lambert, P. C. (2011). Flexible parametric survival analysis using stata: Beyond the Cox model. College Station, TX: StataCorp LP., Stata Press.

Royston, P., \& Parmar, M. K. B. (2002). Flexible parametric proportional-hazards and proportional-odds models for censored survival data, with application to prognostic modelling and estimation of treatment effects. Statistics in Medicine, 21, 2175-2197. doi:10.1002/sim.1203

Schwarz, G. (1978) Estimating the dimension of a model. Annals of Statistics, 6, 461-464. doi:10.1214/aos/1176344136 